\title{
Unraveling groundwater contributions to evapotranspiration in a mountain headwaters: Using eddy covariance to constrain water and energy fluxes in the East River Catchment
}

\author{
Anna Ryken ${ }^{1}$, David Gochis ${ }^{2}$, and Reed Maxwell ${ }^{3}$ \\ ${ }^{1}$ Colorado School of Mines \\ ${ }^{2}$ National Center for Atmospheric Research \\ ${ }^{3}$ Princeton University
}

January 4, 2021

\begin{abstract}
Despite the importance of headwater catchments for western United States' water supply, these regions are often poorly understood, particularly with respect to quantitative understanding of evapotranspiration (ET) fluxes. Heterogeneity of land cover, physiography, and atmospheric patterns in these high-elevation regions lead to difficulty in developing spatially-distributed characterization of ET. As the largest terrestrial water flux behind precipitation, ET represents a significant fraction of the water budget for any watershed. Likewise, groundwater is the largest available freshwater store and has been shown to play a large role in the water balance, even in headwater systems. Using an eddy covariance tower in the East River Catchment, a Colorado River headwaters basin, this study estimates water and energy fluxes in high-elevation, complex systems to better constrain ET estimates and calculate overall water and energy budgets, including losses from groundwater. The eddy covariance method is used to estimate ET from years 2017 through 2019 at a saturated, riparian end-member site. Owing to complexities in near surface atmospheric structure such as stable boundary layers over snowpack and shallow terrain driven flow from surrounding landscape features, energy flux and ET estimates were limited to the warm season when energy closure residuals from the eddycovariance system were reliably less than $30 \%$, a threshold commonly used in eddy covariance energy flux estimation. The resulting ET estimations are useful for constraining water budget estimates at this energy-limited site, which uses groundwater for up to $84 \%$ of ET in the summer months. We also compared East River ET magnitudes and seasonality to two other flux towers (Niwot Ridge, $\mathrm{CO}$ and Valles Caldera, NM), located in the Rocky Mountains. This data is useful for constraining ET estimates in similar end-member locations across the East River Catchment. Our results show that groundwater-fed ET is a significant component of the water balance and groundwater may supply riparian ET even during low-snow years.
\end{abstract}

\section{Hosted file}

UnravelingGroundwater.pdf available at https://authorea.com/users/345910/articles/502335unraveling-groundwater-contributions-to-evapotranspiration-in-a-mountain-headwatersusing-eddy-covariance-to-constrain-water-and-energy-fluxes-in-the-east-river-catchment 

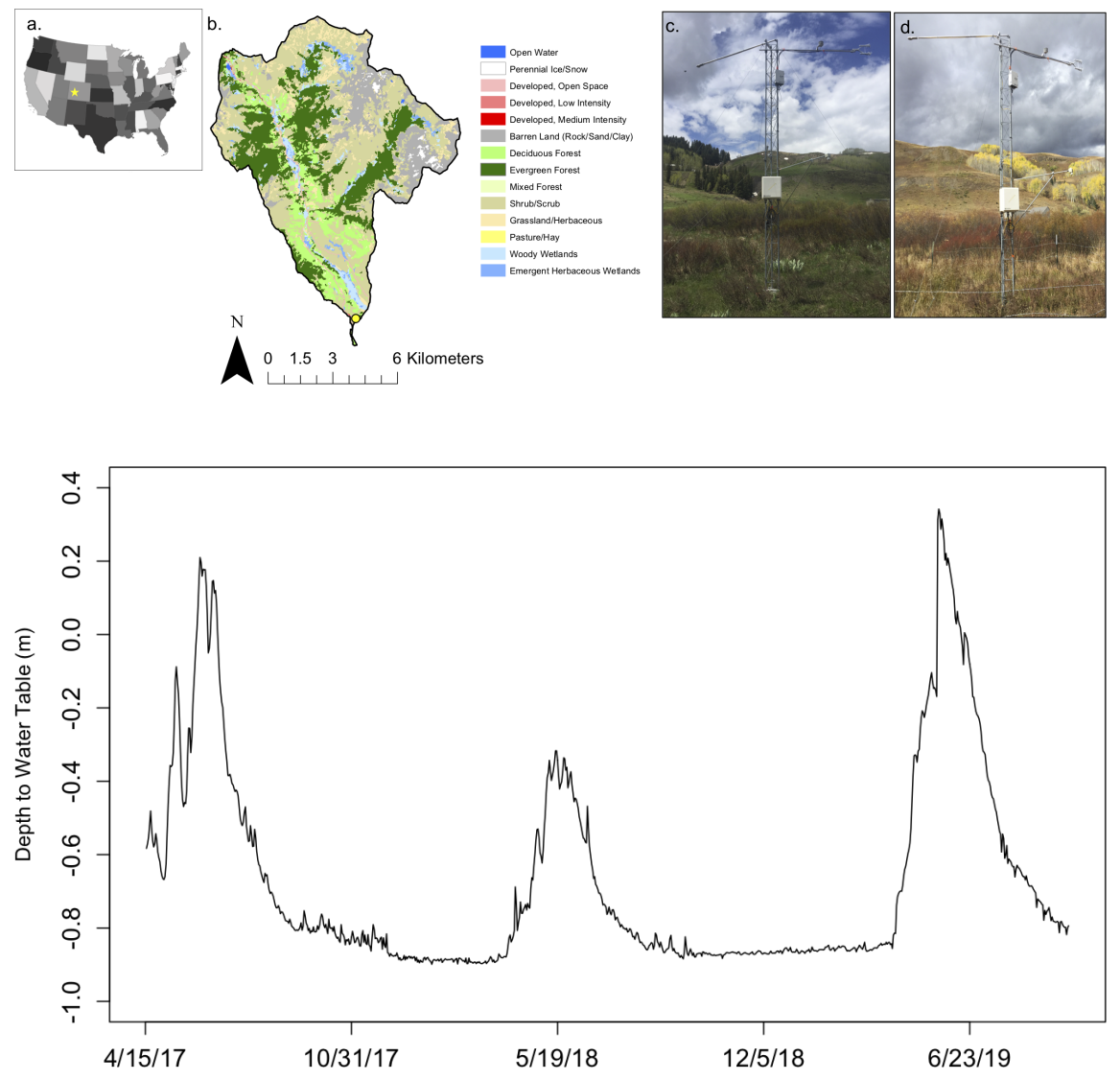

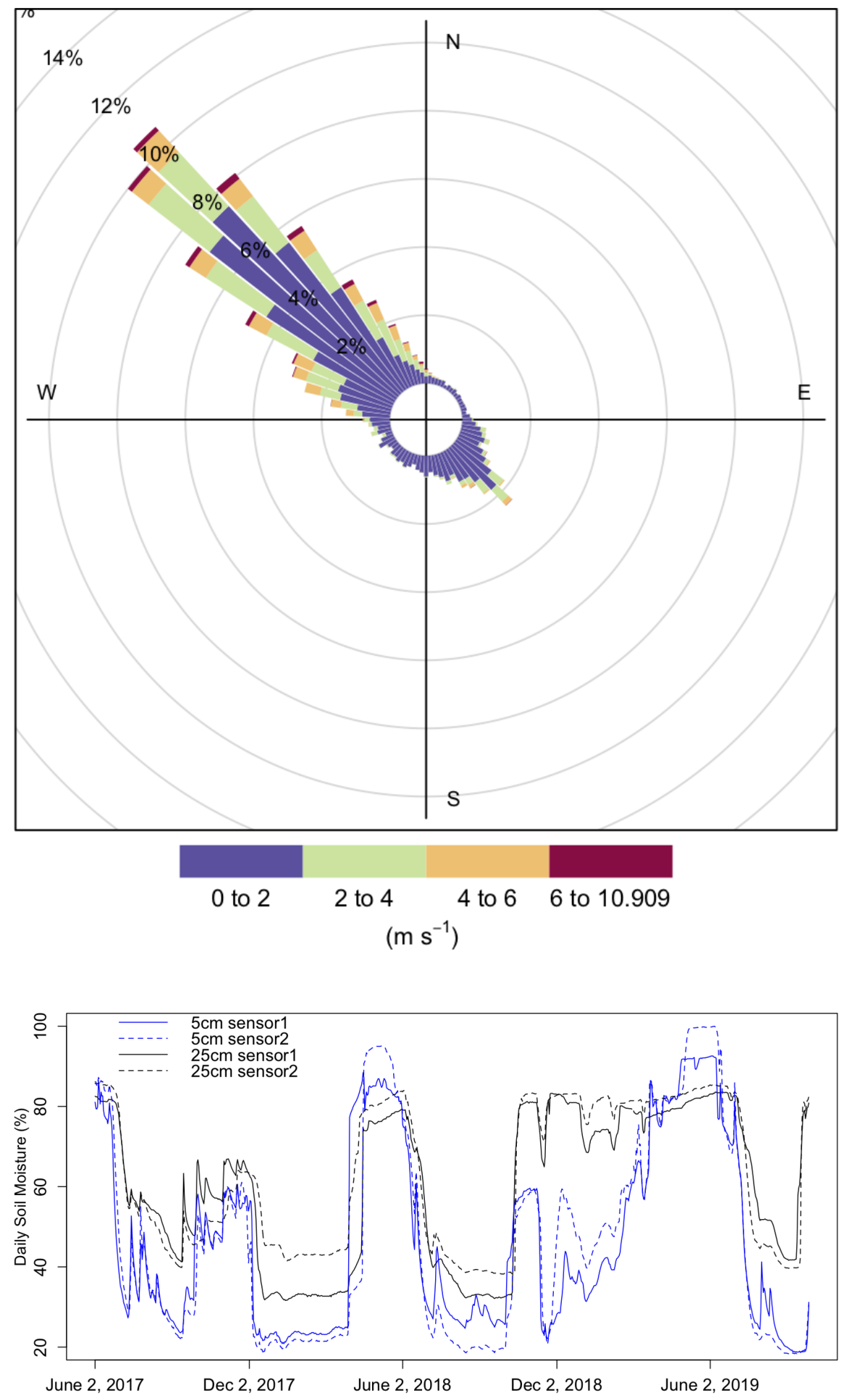

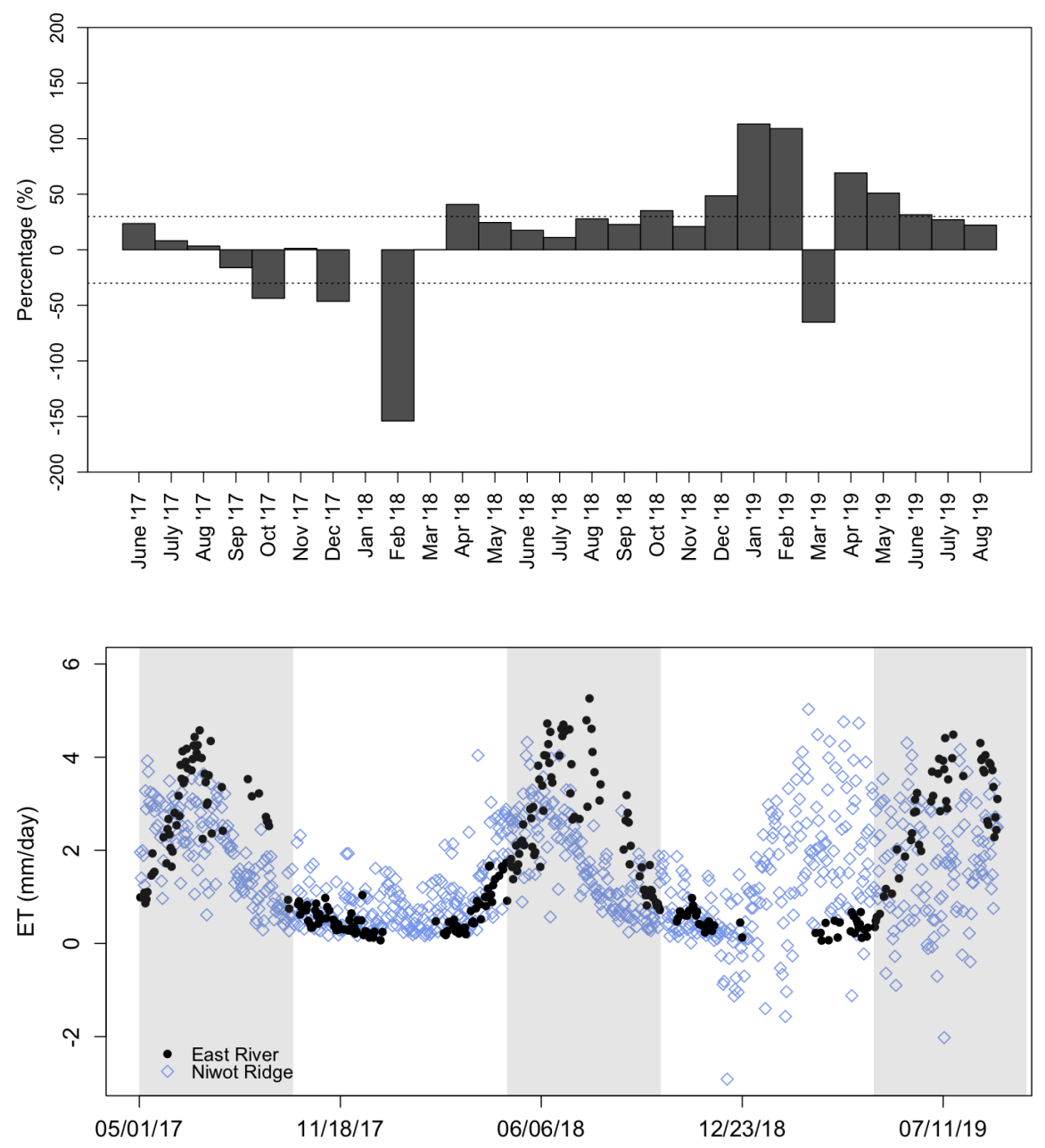

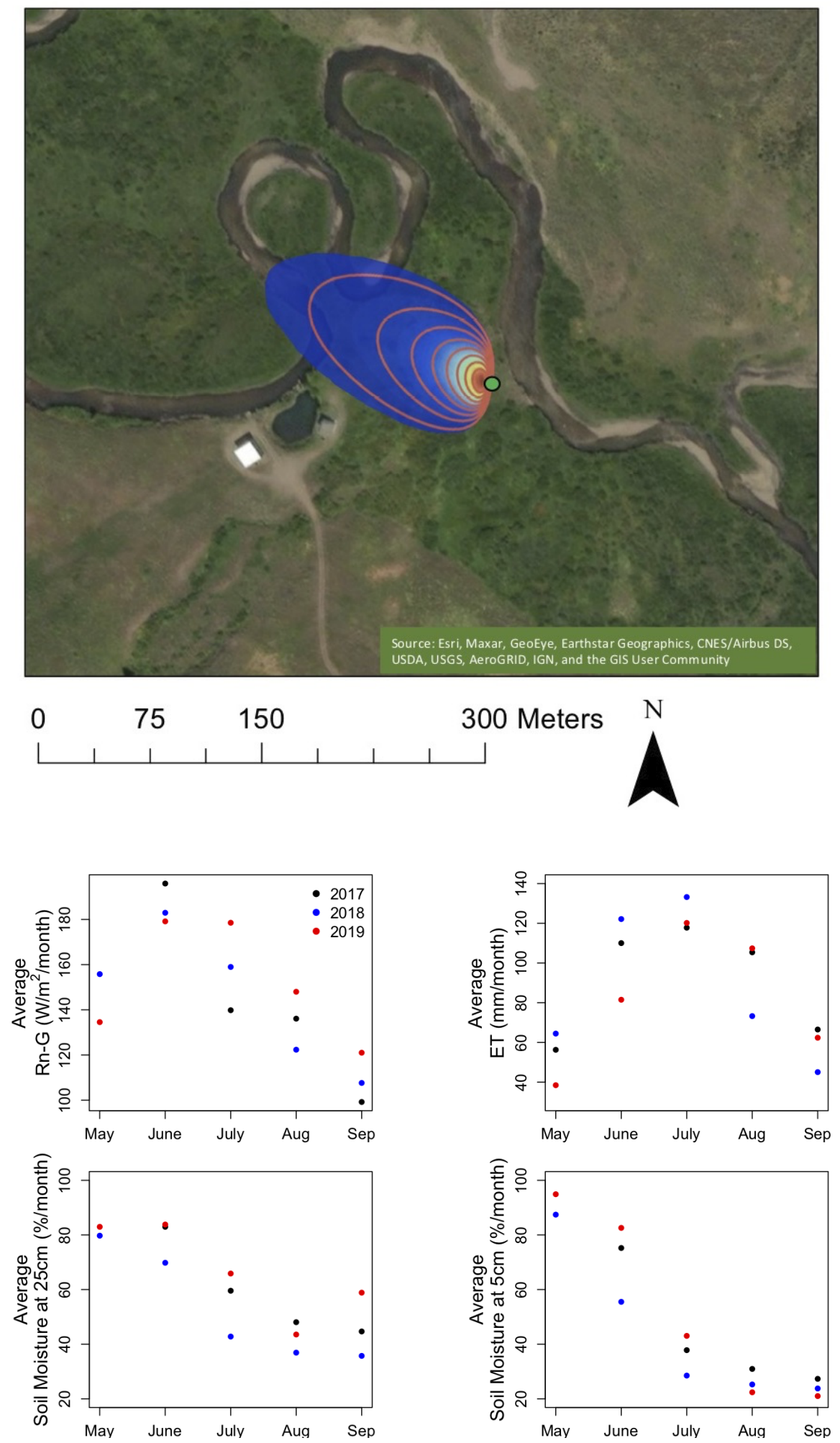

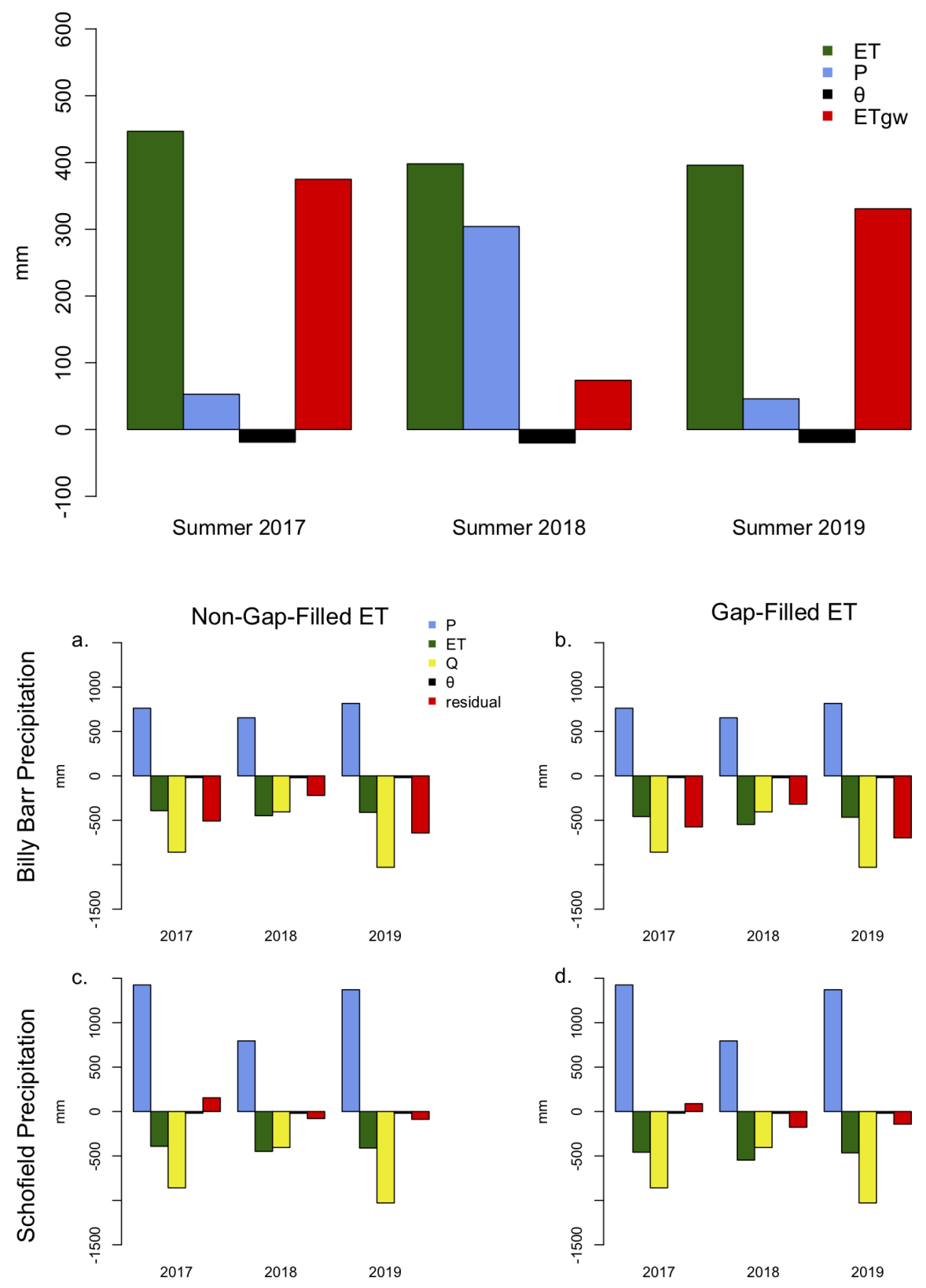


\begin{tabular}{|c|c|c|c|}
\hline $\begin{array}{l}\text { Meteorological } \\
\text { Variable }\end{array}$ & Sensor & Make/Model & $\begin{array}{l}\text { Sampling } \\
\text { Frequency }\end{array}$ \\
\hline Wind Speed & $\begin{array}{l}\text { 3-dimensional sonic } \\
\text { anemometer }\end{array}$ & $\begin{array}{l}\text { Campbell Scientific } \\
\text { IRGASON }\end{array}$ & $10 \mathrm{~Hz}$ \\
\hline Air Temperature & sonic anemometer & $\begin{array}{l}\text { Campbell Scientific } \\
\text { IRGASON }\end{array}$ & $10 \mathrm{~Hz}$ \\
\hline Barometric Pressure & sonic anemometer & $\begin{array}{l}\text { Campbell Scientific } \\
\text { IRGASON }\end{array}$ & $10 \mathrm{~Hz}$ \\
\hline $\begin{array}{l}\text { Water Vapor } \\
\text { Concentration }\end{array}$ & infra-red gas analyzer & $\begin{array}{c}\text { Campbell Scientific } \\
\text { IRGASON }\end{array}$ & $10 \mathrm{~Hz}$ \\
\hline $\begin{array}{c}\mathrm{CO}_{2} \mathrm{Gas} \\
\text { Concentration } \\
\end{array}$ & infra-red gas analyzer & $\begin{array}{l}\text { Campbell Scientific } \\
\text { IRGASON }\end{array}$ & $10 \mathrm{~Hz}$ \\
\hline $\begin{array}{c}\text { Incoming/Outgoing } \\
\text { Shortwave/Longwave } \\
\text { Radiation }\end{array}$ & $\begin{array}{l}\text { ingoing/outgoing } \\
\text { pyranometer } \\
\text { incoming/outgoing } \\
\text { pyrgeometer }\end{array}$ & Kipp and Zonen CNR-4 & 15 minutes \\
\hline Soil Heat Flux & heat flux plate & $\begin{array}{l}\text { Huskeflux self-calibrating } \\
\text { soil heat flux plate }\end{array}$ & 15 minutes \\
\hline Soil Temperature & soil thermistor & Steven's Hydra-Probe & 15 minutes \\
\hline Soil Moisture & soil dielectric probe & Steven's Hydra-Probe & 15 minutes \\
\hline $\begin{array}{c}\text { Soil Electrical } \\
\text { Conductivity }\end{array}$ & $\begin{array}{c}\text { soil electrical } \\
\text { conductivity probe }\end{array}$ & Steven's Hydra-Probe & 15 minutes \\
\hline $\begin{array}{l}\text { Surface Infra-red } \\
\text { Skin Temperature }\end{array}$ & infra-red radiometer & $\begin{array}{c}\text { Apogee SI-111 Infra-red } \\
\text { radiometer }\end{array}$ & 15 minutes \\
\hline & $\begin{array}{c}\text { data logging/control } \\
\text { system }\end{array}$ & Campbell Scientific CR3000 & \\
\hline
\end{tabular}

\begin{tabular}{|l|l|}
\hline Sampling Frequency & $10 \mathrm{~Hz}$ \\
\hline Sensor Orientation & 315 degrees (from North) \\
\hline Measurement Height & 5.9 meters \\
\hline Canopy Height & 2 meters \\
\hline Displacement Height & Diagnosed online \\
\hline Roughness Length & Diagnosed online \\
\hline Site Elevation & 2862 meters ASL \\
\hline Wind Coordinate Rotation & Planar-fit method (Wilczak, Oncley, \& Stage, \\
& $2001)$ \\
\hline $\begin{array}{l}\text { Spectral Correction (low pass filter } \\
\text { correction) }\end{array}$ & Massman (2001) \\
\hline Density Corrections & WPL method (Webb, Pearman, \& Leuning, \\
& $1990)$ \\
\hline
\end{tabular}




\begin{tabular}{|c|c|c|c|}
\hline & $\mathbf{2 0 1 7}$ & $\mathbf{2 0 1 8}$ & $\mathbf{2 0 1 9}$ \\
\hline Latent Heat $\left(\mathrm{W} / \mathrm{m}^{2}\right)$ & 105.24 & 92.65 & 95.55 \\
\hline Summer ET (mm) & 334.48 & 294.49 & 303.69 \\
\hline Annual ET (mm) & $457.94^{\dagger}$ & 547.12 & 465.54 \\
\hline Rn-G (W/m²) & 157.26 & 154.75 & 168.55 \\
\hline Air Temperature (K) & 286.59 & 286.41 & 285.56 \\
\hline Dew Point Depression & 11.42 & 12.13 & 12.04 \\
\hline $\begin{array}{c}\text { Annual Precipitation } \\
(m m)\end{array}$ & 1037.5 & 790.13 & 1115.61 \\
from Billy Barr Station & & & \\
\hline
\end{tabular}

\begin{tabular}{|c|c|c|c|}
\hline & $\mathbf{2 0 1 7}$ & $\mathbf{2 0 1 8}$ & $\mathbf{2 0 1 9}$ \\
\hline ER Summer Evapotranspiration (mm) & 334.48 & 294.49 & 303.69 \\
\hline VC Summer Evapotranspiration (mm) & 398.47 & 381.44 & 453.63 \\
\hline NR Summer Evapotranspiration (mm) & 230.53 & 218.31 & 151.63 \\
\hline ER Annual Evapotranspiration (mm/year) & 457.94 & 547.12 & 465.54 \\
\hline VC Annual Evapotranspiration (mm/year) & 731.35 & 849.79 & 855.64 \\
\hline NR Annual Evapotranspiration (mm/year) & 320.02 & 464.10 & 499.64 \\
\hline ER Temperature (K) & 286.59 & 286.41 & 285.56 \\
\hline VC Temperature (K) & 289.98 & 290.86 & 290.29 \\
\hline NR Temperature (K) & 285.42 & 286.39 & 284.25 \\
\hline ER Precipitation (mm, cumulative) & 1037.5 & 790.13 & 1115.61 \\
\hline VC Precipitation (mm, cumulative) & 539.56 & 299.51 & 545.69 \\
\hline NR Precipitation (mm, cumulative) & 979.52 & 745.57 & 848.10 \\
\hline
\end{tabular}

\begin{tabular}{|c|c|c|c|}
\hline & $\mathbf{2 0 1 7}$ & $\mathbf{2 0 1 8}$ & $\mathbf{2 0 1 9}$ \\
\hline Billy Barr & -507.98 & -220.05 & -643.08 \\
\hline Billy Barr and ET Gap-filled & -574.11 & -318.52 & -697.87 \\
\hline Schofield & 155.13 & -79.10 & -87.60 \\
\hline Schofield and ET Gap-filled & 89.00 & -177.55 & -142.39 \\
\hline
\end{tabular}

